\title{
Applying Bayesian model averaging for uncertainty estimation of input data in energy modelling
}

Monika Culka

\begin{abstract}
Background: Energy scenarios that are used for policy advice have ecological and social impact on society. Policy measures that are based on modelling exercises may lead to far reaching financial and ecological consequences. The purpose of this study is to raise awareness that energy modelling results are accompanied with uncertainties that should be addressed explicitly.

Methods: With view to existing approaches of uncertainty assessment in energy economics and climate science, relevant requirements for an uncertainty assessment are defined. An uncertainty assessment should be explicit, independent of the assessor's expertise, applicable to different models, including subjective quantitative and statistical quantitative aspects, intuitively understandable and be reproducible. Bayesian model averaging for input variables of energy models is discussed as method that satisfies these requirements. A definition of uncertainty based on posterior model probabilities of input variables to energy models is presented.

Results: The main findings are that (1) expert elicitation as predominant assessment method does not satisfy all requirements, (2) Bayesian model averaging for input variable modelling meets the requirements and allows evaluating a vast amount of potentially relevant influences on input variables and (3) posterior model probabilities of input variable models can be translated in uncertainty associated with the input variable.

Conclusions: An uncertainty assessment of energy scenarios is relevant if policy measures are (partially) based on modelling exercises. Potential implications of these findings include that energy scenarios could be associated with uncertainty that is presently neither assessed explicitly nor communicated adequately.
\end{abstract}

Keywords: Uncertainty; Energy modelling; Assessment methods; Bayesian model averaging

\section{Background}

Energy scenarios are quantitative or qualitative output from mathematic models ${ }^{\mathrm{a}}$ of the energy system, or, systematic, consistent thinking in qualitative terms about the energy system. Quantitative energy models can be classified as top-down models, typically macro-economic models with focus on energy economics and bottom-up models, typically technology oriented process-based models. Different mathematical descriptions of the target system are possible, such as general equilibrium models (e.g. E3ME [1]), linear programs (e.g. TIMES [2]), stochastic models (e.g. [3], especially [4]) or mixed complementary problems (e.g. [5]). If an energy model has an objective function to be minimised or maximised, it is an optimisation model. Opposed to these, simulation models simulate consequences over time

Correspondence: monika.culka@kit.edu

KIT Institute of Philosophy, Kaiserstr.12, Karlsruhe 76128, Germany of key assumptions. Frequently used terms in that context are business as usual scenario or reference scenario. An energy scenario can describe both, key assumptions about relevant input variables in energy economics, and the result of a model run, the model output. In this text, the term energy scenario refers to the results of quantitative models. Amongst the most important input variables, also called key assumptions or assumption framework, are for example shares of specific electricity generation capacities, population growth assumptions, fuel price assumptions or gross domestic product assumptions. These key assumptions can be varied to produce alternative scenarios that can be assessed quantitatively by means of energy models or qualitatively in different storylines. The choice which key assumptions are considered in a study strongly depends on the aim of the research and is often coupled to specific questions regarding energy futures or political 
considerations cf. [6,7]. One of the main aims of such energy scenarios are statements about the future, be it possible, probable, normative or deterministic statements. These statements may serve for political advice, or are the basis for (energy-) political decision making. Examples to illustrate this function are the Energiekonzept der Bundesregierung [8] in Germany that is - at least inspired by a modelling exercise, or, on a European level, the Energy Roadmap 2050 [9] that refers to a modelling exercise, detailed in part $2 / 2$ of their publication.

The scenario report for the Energiekonzept introduces its findings with a clarification on what energy scenarios, as described in the report, are meant to be: 'Scenarios describe possible futures. They do not claim to represent the most likely development from today's perspective. [...] Depending on the definition of relevant parameters ('Eckpunkte'), next to the derived scenarios, many other pathways in the future of the German energy supply are possible that are not under scrutiny in this work' [10].

So, what discerns such a possibilistic statement (an energy scenario) from any other possibilistic energy scenario that, admittedly, may serve the same purpose of fulfilling the German energy supply? And if there is no difference, of what profit is the modelling exercise altogether? One answer can be found in the document, the developed target scenarios comprise consistent pathways of long-term energy economic developments [10].

The question arises, what consistent in this context means. Consistent with expectations about the future, consistent with past evidence, consistent with the mathematical model framework or consistent in a rather abstract sense that there are no contradictions in the energy scenarios. However interpreted, the question remains, what additional value, with respect to any other possible pathway, does a model-based pathway into the German energy future provide? Consistency, in the broadest sense could be understood in and by itself as possibility; for, if a statement is self-contradictory, it is not possible. Hence, consistency is no unique characteristic of possible model-based statements. However, it is possible that some energy scenarios are not even consistent in a non-contradictory sense. Weimer-Jehle has developed a systematic approach to ensure that assumption preselection and associated effects on society, economy and environment are consistent and evaluated in a transparent way [11]. If such a transparent approach in the phase of key assumption selection or scenario construction is not provided, it remains at least questionable, if energy scenarios are not self-contradictory.

An added value could be derived, if model-based energy scenarios were contrasted to other possible scenarios, particularly, if an uncertainty assessment was carried out for the energy scenario. In the case of a quantitative uncertainty assessment, the possibility of comparing different scenarios with respect to their adherent uncertainty could indicate to what extent and in what respect the scenario can be discerned from other scenarios. An energy scenario that is possible begs the question how possible it is, given key assumptions about the future. The question is thus, how uncertain is an energy scenario?

The main objective of this text is a discussion which requirements an uncertainty assessment should fulfil. Based on these requirements, a method, Bayesian model averaging (BMA) is debated as possible candidate to satisfy them. The main arguments are that an energy modelling exercise that is enriched with an uncertainty assessment which satisfies the requirements is (1) comprehensible in terms of its associated uncertainty and (2) should contribute to a complete understanding of energy scenarios, especially if they are used in decision support.

Uncertainties in energy scenarios can have different sources and can be of different kinds. Walker et al. have presented a concise summary of existent uncertainty of model-based energy scenarios, based on the location, level and nature of the uncertainty [12]. According to them, generic locations can be context, model uncertainty, inputs, parameter and outcome. The uncertainty estimation method discussed in this text, BMA for input variables to energy models, can assess uncertainty in the location input and parameter uncertainty, and, to an extent, context uncertainty that concerns the modelled boundaries of the system. The presented method does not aim to evaluate error propagation within a specific energy model. Walker describes the level of uncertainty in terms of determinism, statistical uncertainty, scenario uncertainty, recognised ignorance and indeterminacy, i.e. total ignorance. BMA for input variables to energy models represents uncertainty based on probabilistic assessment and hence can also range from certainty to total ignorance. However, the use of statistical data renders the assessment itself prone to statistical uncertainty. Finally, Walker et al. describe the nature of uncertainty as epistemic uncertainty or variability where epistemic uncertainty is due to the imperfection of our knowledge and variability refers to inherent variability, especially present in human and natural systems. The BMA approach can evaluate both natures of uncertainty in statistical terms. If input variables to energy models are exposed to variability, data fit of model results with respect to statistical data will indicate that exposure.

Uncertainty due to variability can also be addressed by stochastic modelling. A stochastic modelling approach aims to represent (natural) variability within the model, e.g. $[13,14]$. Such uncertainty analyses are mainly applicable in physical systems. Energy models that also represent economic, political, environmental and social aspects of an energy system regard the system from a broader perspective. However, stochastic uncertainty assessments could be beneficial for (parts of) energy models that are 
exposed to variability such as electricity generation modelling based on wind or solar cf. $[15,16]$. Epistemic uncertainty is less naturally defined in a probabilistic framework, and hence, one of the objectives of uncertainty quantification is the reformulation of epistemic uncertainty as variability [17]. If epistemic uncertainty is contained in the energy model, the model results are likely to be more uncertain than the input variables. The BMA approach accounts for that by establishing a lower bound of uncertainty.

Recent evaluations have investigated the nature of energy scenarios and their limitations in terms of legitimate inference from model output [6]. In contrast to a vivid discussion of model quality and legitimate inference, as can be observed in climate modelling [18-21], energy models have not invoked a similar discussion. Whilst climate modelling developed a framework for the treatment and communication of uncertainties [22-24], energy models and resulting scenarios lack such a systematic approach for uncertainty qualification (or quantification). However, investigating uncertainties in models is necessary for quality assessment of model results and reliability of results, especially if such results figure in policy advice.

The next chapter will investigate existing uncertainty assessments in energy modelling and focus on the strengths and weaknesses of those. From these considerations, general requirements that an uncertainty assessment for energy modelling should satisfy are retrieved. In the following section, presently applied methods for uncertainty evaluation are discussed, including expert elicitation, robustness analysis, model fit, variety of evidence and standard statistical analysis. As research regarding uncertainty evaluation for energy models is not yet as advanced as for example in climate science, a substantial part of discussion is based on examples of other disciplines, especially climate science. The next two sections firstly address critique on Bayesian approaches and present the uncertainty assessment based on BMA for input variables of energy models. The last section summarises results and discusses the method critically.

\section{Existing uncertainty assessments in energy modelling}

Data of already existing publications are compared with the results of this work, such that an evaluation of existing approaches to uncertainty assessment in the context of energy economics is examined on the basis of two examples.

Walker et al. [12] have investigated energy model-related uncertainties with respect to their nature and occurrence. Their definition of uncertainty being 'any departure from the unachievable ideal of complete determinism, allows for a conceptual investigation of all relevant uncertainties, by defined categories, reaching from determinism to total ignorance. The provided tool, an uncertainty matrix, should be used to identify model outcome uncertainty according to their level and nature. It is not clear in what terms the matrix should be evaluated, yes/no, much/little, or, in a numeric scale that is not provided. This approach allows for an illustrative representation of uncertainties involved in modelling. However, the method seems to end with a delicate categorization rather than with a valuable assessment. Indeed, awareness of the location, level and nature of uncertainty is important information; nonetheless, the method does not provide insight into how the uncertainty should be assessed or into what way uncertainty of, for example, recognised ignorance in the location model structure bears on the uncertainty of model outcomes.

The second example of an assessment is the numeral unit spread assessment pedigree (NUSAP) method to assess qualitative and quantitative uncertainties in the targets image energy model regional (TIMER) energy model, part of RIVMs IMAGE model [25]. Firstly, by means of a comprehensive checklist for model quality assurance, key loci and sorts of uncertainties in the TIMER modelling process are identified. Model structure uncertainties were analysed by a meta-analysis of similarities and differences of six energy models. A sensitivity analysis for model parameters in terms of magnitude of influence has been carried out. A NUSAP expert elicitation workshop has systematically assessed those parameters in the following dimensions: proxy, empirical basis, theoretical understanding, methodological rigour and validation. And finally, a diagnostic diagram is produced [25].

This evaluation provides interesting insight in the TIMER model, the uncertainties associated with the modelling process and the model results. However, there is some critique that relates to the applicability and required expert knowledge for such an assessment. Firstly, the method is rather model-specific. Secondly, the method relies mainly on expert elicitation for both experts in modelling and energy economics. Experts in modelling are likely to be experts for especially the model they work with. Large and complex models tend to require long periods of vocational adjustment before a model is fully understood. And due to intellectual property considerations, some models cannot be assessed by 'foreign' modellers. Another critical remark considers the output of the assessment. The diagnostic diagram, as presented in section 6.8 of document [25], is difficult to understand. The diagnostic diagram is based on the results of expert elicitation.

Three groups have evaluated the same parameter, e.g. $\mathrm{N}^{\circ}$ 8a learning rates for nuclear. For a discussion of an expert elicitation on future costs of nuclear, Rai provides interesting insight [26]. The resulted diagram of the expert elicitation is illustrated in Figures 1,2 and $3^{\mathrm{b}}$ [25]. The figures show a kite chart that consist of six spokes 


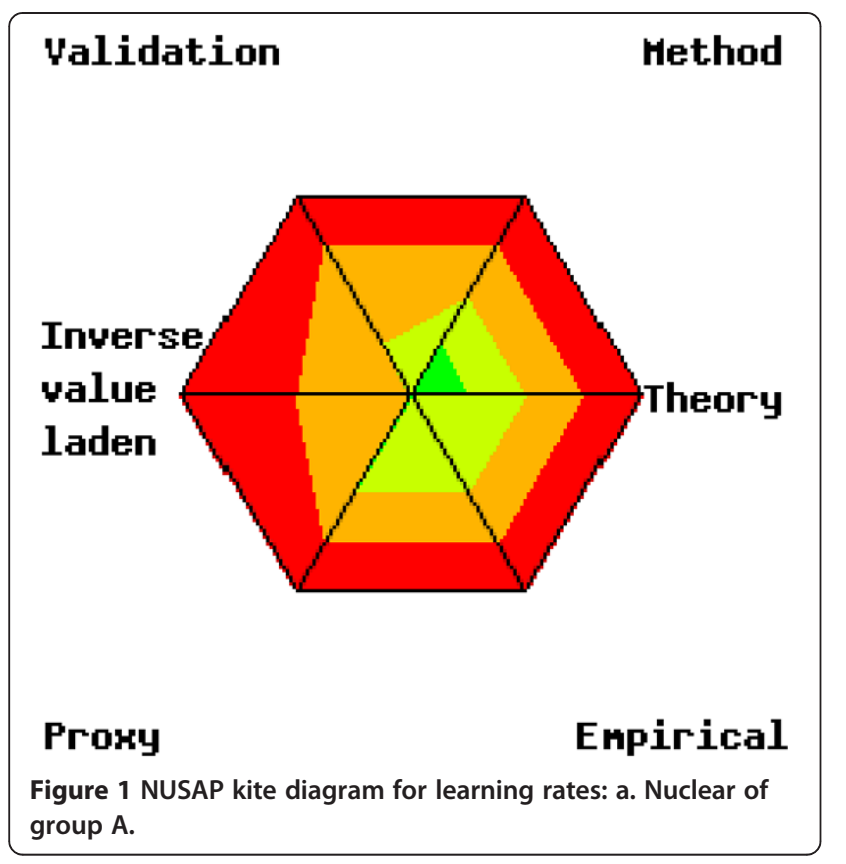

representing the dimensions of evaluation, namely, validation, method, theory, empirical, proxy and inverse laden value. The green kite is spanned up by the minimum scores in each group for each pedigree criterion; the orange kite is spanned up by the maximum scores. The orange band between the green kite and the red area represents expert disagreement on the pedigree scores for that variable. In some cases, the picture was strongly influenced by a single deviating low score given by one of the six experts. In those cases, the light green kite

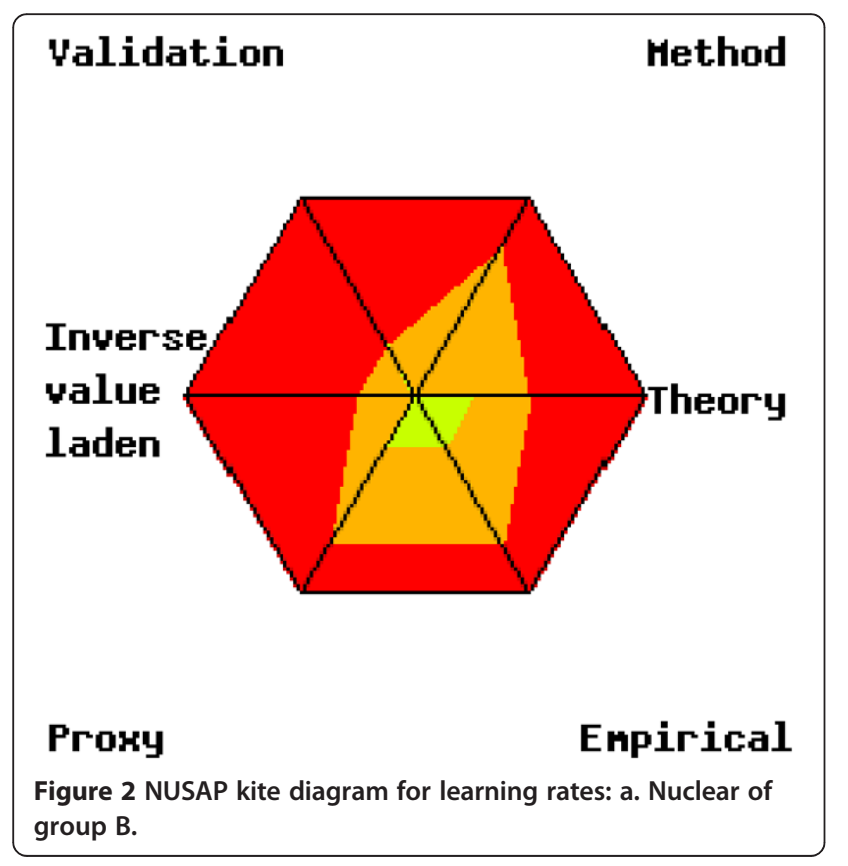

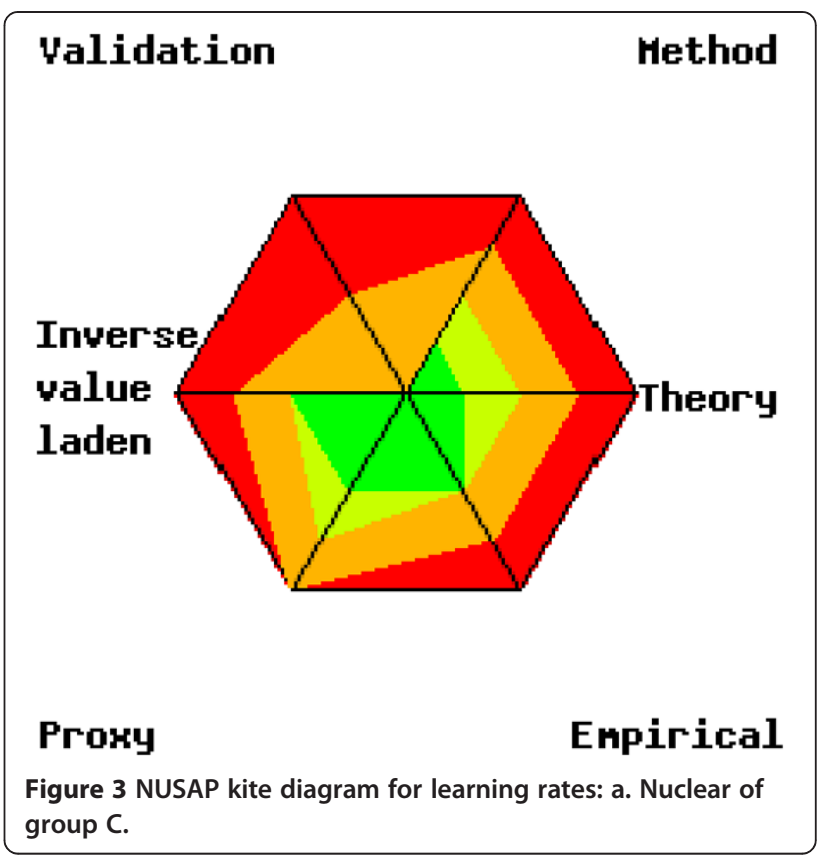

shows what the green kite would look like if that outlier would have been omitted. The 0 is in the center of the diagram and the 4 is on each corner. Note that, the scores for value ladenness have been inverted compared to what was filled in on the cards: a 4 on card was entered as a 0 in the diagram and a 0 on the card as a 4 in the diagram.

These three evaluations all represent the uncertainty associated with the learning rates of nuclear power production. There are several interpretations possible: either, the dissent in the expert groups indicates that the experts do not dispose of deep understanding of the question ${ }^{\mathrm{c}}$.

Or, one expert group is correct and the others are wrong. Or, the limited number of experts does not allow for the results to converge to an unambiguous assessment result. Or, the strong dissent indicates that uncertainty is high. This last reading is not without further ado more justified than any of the other readings. Yet, it is the only one that actually assesses the uncertainty of the parameter in question. This example illustrates the necessity of an inter-subjective requirement in an assessment method. An inter-subjective assessment method would render the result of an uncertainty assessment less dependent on the specific individuals that carry out the analysis. If an assessment is based on expert elicitation and possible interpretations of dissent (and consent) are not explicit, the method itself contributes to uncertainty. For then, the uncertainty of the assessment method itself and the uncertainty of the model assessed are present, and it can be difficult to cleave them apart. It is necessary to stress that expert elicitation is an important tool. However, due to practical limitations ${ }^{\mathrm{d}}$, issues of the method in and 
by itself, such as convergence in findings and trustworthiness of findings, need to be addressed and evaluated.

Based on these examples and general desirables for an uncertainty assessment, a list of requirements of an uncertainty assessment for energy scenarios can be formulated. The six following requirements are not exhaustive; other possibly relevant virtues could be imagined as requirements for uncertainty quantification are context dependent. The presented aspects are inspired by existing approaches and desirables with respect to methodology and practical aspects in energy economics and climate science. An uncertainty assessment should

1) give a clear indication how reliable the findings are (uncertainty assessment)

2) be applicable independent of assessor's expertise (inter-subjectivity)

3) be applicable to different models (comparability of results)

4) incorporate qualitative and quantitative aspects (complete representation)

5) be intuitively understandable and straightforward to communicate (scale requirements)

6) be reproducible and unambiguous.

A clear indication of the reliability of findings (requirement 1) can be achieved by quantitative methods rather than qualitative methods. Classical uncertainty quantification methods include, but are not limited to, both Frequentist and Bayesian statistical analysis, stochastic models, sensitivity analysis, or Monte Carlo methods $[17,27]$. Which quantification method is used strongly depends on the target system under scrutiny, the model representation of that system and the aspired preciseness of the uncertainty quantification. Inter-subjectivity (requirement 2) can be interpreted as a maximisation of objectiveness in the assessment process. However, the incorporation of both quantitative and qualitative aspects (requirement 4), does implicitly demand subjective characteristics, for qualitative assessment methods demand subjective evaluation and understanding. The requirement of intuitive comprehensibility (requirement 5) should allow the recipient of such assessments an interpretation without tedious lecture of explanatory notes. And finally, reproducibility and unambiguousness (requirement 6) should minimise misunderstandings and increase confidence in the evaluation by the recipient $\mathrm{e}^{\mathrm{e}}$. The expected scientific progress by the proposed BMA method of uncertainty assessment in the context of energy economics can be summarised as follows

- The assessment method does not solely rely on expert elicitation, although valuable subjective expert knowledge can be included.
- BMA could provide a versatile tool for the assessment of complex interrelated statistical data.

- Requirements that should be satisfied by an uncertainty measurement method are met.

An explicit uncertainty assessment of energy scenarios that satisfies these requirements would increase transparency of assumption uncertainty and thus model results. The aims of the text are to present a methodology, BMA for input variables of energy models, that satisfies these requirements and to infer quantitative uncertainty estimations from input parameters to energy models. Recipients of energy scenarios could gain a better understanding regarding the uncertainty of model results (i.e. energy scenarios) what might impact their function as decision support or basis for decision, especially if energy scenarios are used for policy advice, leading to far reaching ecological, financial and societal consequences.

\section{Methods \\ Quantitative methods versus qualitative methods for uncertainty assessments}

In the quest of an appropriate uncertainty assessment for energy scenarios, climate science may provide a suitable starting point as the uncertainty assessment discussion in climate sciences is more advanced than in energy economics.

The Intergovernmental Panel on Climate Change (IPCC) has developed over years guidelines for a consistent treatment of uncertainties associated with climate modelling results. For the current report, the fifth assessment report, the published guidelines incorporate many of former critics on the initial uncertainty assessments and critically analyses uncertainty assessments of earlier reports [28]. The guidelines of the assessment report 5 (AR5) specify two metrics for the communication of the degree of certainty in key findings:

- Confidence in the validity of a finding, based on the type, amount, quality and consistency of evidence (e.g. mechanistic understanding, theory, data, models, expert judgement) and the degree of agreement. Confidence is expressed qualitatively.

- Quantified measures of uncertainty in a finding expressed probabilistically (based on statistical analysis of observations or model results, or expert judgement) [23].

By means of a confidence matrix, a likelihood scale (expressed as probabilities) and probability distribution functions, the three working groups of the AR5 are to evaluate uncertainties associated with their findings. The likelihood scale for (subjective) quantitative assessment 
of uncertainty is recommended to be applied only in cases with high or very high confidence [29] $]^{\mathrm{f}}$.

The IPCC uncertainty assessment thus relies on both, qualitative and quantitative ways to describe reliability of findings. Apparently, if quantitative assessments are applicable, they should be used preferable to qualitative assessment. Qualitative uncertainty assessment is applied in cases of deep uncertainty ${ }^{\mathrm{g}}$, where uncertainty cannot be quantified. Qualitative uncertainty assessment faces several challenges. The problem of linguistic ambiguity seems to be the predominant problem when uncertainty is qualitatively assessed. In the guidance, note the level of confidence is defined using five qualifiers: very low, low, medium, high and very high [23]. It synthesises the author teams' judgments about the validity of findings as determined through evaluation of evidence and agreement. It is arguable if there is a common understanding of such categories amongst individuals and hence, the question arises, whether the evaluation of agreement actually depicts the uncertainty of the finding in question or rather the ambiguity in understanding of the term used. Also, there is no clear indication how much agreement is necessary for the affiliation to a certain category ${ }^{\mathrm{h}}$. And, finally, it is unclear in which way agreement can be associated with high confidence and in turn with uncertainty (judgments about the validity of findings). One interpretation could be that high confidence (inter alia based on high agreement) means low uncertainty; however, this could not hold true in cases where agreement is high that the level of uncertainty is high for a finding (e.g. due to the stochastic nature of a process). Moreover, this reading also faces the criticism that it is thinkable that even with high agreement, the finding is not at all certain, and all assessors could collectively be wrong in their valuation. The other reading, that high confidence means high uncertainty, next to being counterintuitive, does not reflect that agreement sometimes does give an indication for the truth of a finding.

Qualitative assessment methods, even if normalised to summary terms (IPCC) seem to intrinsically depend on not only a subjective comprehension of summary terms but also subjective opinion of the assessor. This can be advantageous or disadvantageous, depending on the expertise of the assessor and the communication of relevant information that influenced the assessment. In any case, such assessment methods lack the important property of generating reproducible assessments. If a different group of experts assessed the results, the uncertainty assessment of a specific finding might turn out to be significantly different, even if a sound reasoning underpins the assessment. As Krueger et al. point out, expert opinion in modelling will benefit from formal, systematic and transparent procedures [30]. Inter-subjective reproducibility is a necessity if a finding is called robust. A qualitative assessment is likely to be not as efficient in evaluating robustness as a quantitative, standardised assessment, given the problem of linguistic ambiguity and subjectivity of the assessment method. A quantitative approach that uses a method that can be standardised and applied independent of the expertise of the assessor would presumably yield higher agreement.

However, qualitative uncertainty assessments have the important benefit of putting findings into perspective of the state of art of modelling and the present knowledge about processes and/or assumptions. If a finding is based on limited knowledge, it cannot represent a certain statement and has to be supplemented with information regarding the validity of findings.

Quantitative assessment methods often face the critique of being perceived with more precision than justified [31,32], especially [33] when he discusses Nowotny's perspective. This could be the case where probability density functions (pdfs) can be produced but are themselves based on uncertain input. In such cases, communication (qualitatively or quantitatively) of the uncertainty related to the pdfs is necessary. An advantage of quantitative methods is an unambiguous representation. The intuitive understanding, even in the simplest form, for example, a scale from one to ten, ten representing high uncertainty, might allow the recipient of such an assessment a clear understanding. This is, indeed, not unproblematic. For one, there is an intrinsic assumption that must be clarified if not true, which is that the scale units are uniform in size ${ }^{\mathrm{i}}$ or a logarithmic scale. Even more intuitively understandable appears to be a probabilistic statement. However, regarding the perception of probabilistic uncertainty assessments, Patt et al. report that changes of equal magnitude in assessed probabilities can have different effects in decision-making experiments. For example, a change of 10 percentage points from $90 \%$ to $100 \%$ impacts choices of test persons differently than a change from $50 \%$ to $60 \%$ [34]. Nonetheless, a probabilistic statement is in itself less susceptible to interpretational errors or misunderstandings than a qualitative statement that uses again - words for interpretation that might be ambiguous.

Another relevant advantage of quantified assessments is the simplicity and comparability of results. The benefits of retrieving a result that can be compared to results, say, some years ago are obvious: applying the same quantitative method can be used to evaluate scientific progress in modelling and scientific understanding (if uncertainty decreases) or illustrate that the nature of a process is more complex than assumed years ago (if uncertainty increases). However, a critique formulated by Kandlikar et al. [35] is that biases can result if simple schemes that attempt to represent uncertainty in a uniform manner across many different contexts are depending on how much detail is presented in the information. This effect is 
analysed by ignorance aversion. Indeed, quantification, or for that matter qualification to summary terms, can result in a loss of detail and reasoning. It is not totally clear how bias can be created through such a process. However, it is the very task of an uncertainty assessment to transform information of various kinds (quantitative, qualitative, narrative, implicit assumptions, etc.) into a form that can be understood without the profound expertise that is necessary to accomplish the uncertainty assessment itself.

The question whether a quantitative assessment method is preferable to a qualitative assessment method cannot be answered purely by evaluating the respective (dis-) advantages. There are practical limitations that may render a quantitative assessment impossible. However, for the development of an uncertainty assessment in the context of energy economics, relevant differences to climate science prevail that might justify the preferable use of quantitative methods.

\section{Climate science and energy economics}

The discussion concerning confirmation of climate models may serve as orientation, and energy model evaluation could profit from these considerations. Lloyd [20] concludes that climate models should not be judged primarily or solely on the basis of what they are weak at. This is an important aspect to remember when evaluating energy models as well. Generally, her approach to confirmation 'takes it as a matter of degree; models can accrue credit and trustworthiness upon being supported by empirical evidence as well as by theoretical derivation'. Lloyd illustrates the strengths in terms of confirmation as model fit, variety of evidence, independent support for aspects of the models and robustness for climate models. These concepts, bearing on the reliability of models, can be applied to energy models as well.

\section{Model fit}

Model fit refers to the ability of model results to represent data that can be observed empirically, possibly ex post. Unfortunately, energy models have a rather poor history of model fit [36,37]. Analysis of the main reasons for deviations of model results to evidence are summarised as:

- unanticipated strong political decisions such as closing of mines in the UK, feed-in tariffs in Germany and world climate change concerns;

- unexpected energy requirements, like the transport behaviour and the rush for gas;

- definition and availability of statistical data [36].

The main difference when comparing climate models with energy models is that energy models represent and simulate a well-understood system with mainly economic drivers. In contrast, climate modelling has its challenges in representing chaotic systems with at least partially little understood causal relationships and magnitudes of impact of system components. It was, at least in principle, possible to know today with sufficient accuracy how the energy system will look like in a given point of time in the future. The problem is that many interests must be met and decisions not tend to be of durable nature as political, environmental or economic circumstances change. This is one reason why energy roadmaps, energy strategies and energy programs on a political level are important. These commitments to a specific system state in future allow for energy modellers to accordingly define constraints in models and consequently investigate - using models - different paths to meet the desiderata. Results of such model simulations may be cost-effective, environmental-friendly, socially accepted or other (possibly optimised) system development paths. The reason why model fit of energy models yields a poor record in the past hence is not (primarily) due to little understanding of the system but must rather be contributed to influences on the system of radical nature that cannot be anticipated. Moreover, such radical impacts (e.g. political reorientation) do not lead to any improvement of energy models or target system understanding for their nature is vested in societal decisions that can and should not be anticipated, hence allowing evolvement of society.

\section{Variety of evidence}

Lloyd refers in her analysis to the fact that climate models can accurately predict other variables than, for example, global mean temperature. This type of confirmation translated to energy models could be interpreted as correctly predicted installed capacity for electricity generation, fuel mix and the like. Variety of evidence in energy models has close relation to the constraints and assumptions built into the model. As Knutti et al. [38] state, it is due to the physical principles known to be true, such as conservation of mass, energy and momentum, that can be applied and transferred across hierarchies of models that confidence in climate models is justified. This physical nature of climate modelling can only partially be applied in energy models. Energy systems have physical limitations, e.g. land use, maximum solar radiation or exhaustible resources; however, the system state is mainly dependent on economic drivers, law requirements and incentive policy. These are not physical, law-obeying mechanisms, although, a kind of cause-and-effect relation can be observed. Due to this different nature of the modelled system, variety of evidence cannot be applied for confirmation in the same sense as climate models are verified by true evidence. The same is true for independent support for aspects. 


\section{Robustness}

Lloyd applies a robustness analysis developed by Weisberg [39] that puts forward a robust theorem of the general form: ceteris paribus, if [common causal structure] obtains, then [robust property] will obtain. The causal structure captured in the respective models seems to be the key difference between climate models and energy models. Causal structures in energy models, depending on the model in question, are for example, inverse supply functions [2], whereas in climate modelling, for example, thermodynamic laws are applied ${ }^{j}$ [40]. It seems that climate science partially due to ignorance of (components of) the target system face epistemic uncertainty. Stevens and Bony [41] analyse that for example, tropical precipitation over land and consequently vegetation dynamics are poorly understood. As a result the understanding of the carbon cycle is limited.

It is necessary to clarify the applied interpretation of implication used to analyse Weisberg's theorem. Let A denote the antecedent 'common causal structure' and B denote the consequent 'common property' of the theorem. The 'if...then' clause can be interpreted in different ways. A strict material implication in its truth functional sense means that A is false or B is true [42]. Another interpretation would be a logical implication to state that B is already logically implicit in A. This interpretation means that it is a logical consequence that a common causal structure implies a common property to obtain (ceteris paribus). Another interpretation of implication (A implies $\mathrm{B}$ ) is that $\mathrm{B}$ is deducible from $\mathrm{A}$ by logical reasoning. To prove that it is logically deducible that a common property obtains if a common causal structure obtains would surpass the scope of this text. But, it may well be possible to do so. Weisberg departs in this question from Levins, Orzack and Sober and clarifies that robustness analysis is effective at identifying robust theorems, and, whilst it is not itself a confirmation procedure, robust theorems are likely to be true [39]. It is important that a theorem as put forward by Weisberg does not presuppose the truth of A. In other words, the theorem does not claim to guarantee that if a common causal structure obtains, this implies that a robust property will obtain (ceteris paribus). In this sense, the theorem is much weaker than one would wish for an uncertainty analysis. If robust theorems according to Weisberg are likely to be true, the only case that is unlikely is the one where A is true and B is false, for this renders the theorem to be false. Hence, the unlikely case is that if common causal structure obtains then robust property will not obtain, ceteris paribus. But as indicated by the example of Stevens and Bony, the antecedent 'common causal structure' (A) can well be false. The use of Weisberg's theorem does not indicate if $B$ (robust property will obtain) is true or false if $A$ is false.
Hence, if common causal structure changes in climate models due to new understanding, robustness, defined as such, does not allow inference to the truth of the associated robust property or uncertainty.

In the case of energy models, causal structures face less uncertainty of epistemic nature, but rather uncertainty due to social or political under-determinism of future developments. In this case, robustness could indicate some degree of certainty. However, it is not straightforward to conclude from robust results to uncertainty, even in a qualitative manner.

Another challenging issue in this respect is the ceteris paribus clause. A common approach for robustness analysis is scenario technique. The choice of parameters that are defined stable (ceteris paribus) and parameters or constraints that are varied significantly influences the results of energy models. It is therefore a choice, what results appear robust, for any result could be in principle produced by choice of parameters (e.g. by technology prices in cost optimization models). Hence, robustness as an indicator for uncertainty in energy scenarios has limited potential for uncertainty assessment of energy model results.

\section{A discussion of Bayesian approaches}

Probabilistic interpretation of uncertainty assessments is considered valuable, as the IPCC guidance note for treatment of uncertainties specifies [23]. Uncertainty and risk are to be assessed to the extent possible, and if appropriate probabilistic information is available, special attention to high-consequence outcomes should be given.

Probabilistic uncertainty assessments satisfy requirements 1 (clear indication how reliable the findings are), 5 (intuitively understandable and straightforward to communicate) and 6 (reproducible and unambiguous), if the methodology of assessment is a standardised process. As well in the IPCC guideline notes, as in the approach by Walker,as in the NUSAP method statistical knowledge is considered as knowledge with little inherent uncertainty. It seems thus appropriate to consider an assessment method that is based on statistical data and produces probabilistic uncertainty assessment results.

Bayesian statistics could provide such a method. As Bernardo [43] points out, the comprehension of probability in Bayesian statistics corresponds precisely to the sense in which this word is used in everyday language. This quality corresponds to satisfying requirement 5 (intuitively understandable and straightforward to communicate): the understanding of probability as a conditional measure of uncertainty associated with the occurrence of a particular even, given the available information and accepted assumptions. Bernardo stresses that a conditional probability measure is dependent on two arguments, the event $\mathrm{E}$ with the uncertainty to be measured and the 
conditions $\mathrm{C}$ of the measurement, 'absolute' probabilities do not exist [43].

In typical applications, one is interested in the probability of some event $E$ given the available data $D$, the set of assumptions A which one is prepared to make about the mechanism which has generated the data, and the relevant contextual knowledge $\mathrm{K}$ which might be available. Thus, $\operatorname{Pr}(E \mid D, A, K)$ is to be interpreted as a measure of (presumably rational) belief in the occurrence of the event $\mathrm{E}$, given data $\mathrm{D}$, assumptions $\mathrm{A}$ and any other available knowledge $\mathrm{K}$, as a measure of how "likely" is the occurrence of $\mathrm{E}$ in these conditions [43].

In Bayesian statistics, a prior probability that represents the presumption of the statistician is combined with empirical data to derive a posterior probability by means of Bayes' theorem.

$$
p(\omega \mid D, A, K)=\frac{p(D \mid \omega) p(\omega \mid K)}{\int_{\Omega} p(D \mid \omega) p(\omega \mid K) d \omega}
$$

With $p(D \mid \omega)$ being a formal probability model for some (unknown) value of $\omega$, the probabilistic mechanism which has generated the observed data $\mathrm{D} ; p(\omega \mid K)$ being the prior probability distribution over the sample space $\Omega$, describing the available (expert) knowledge $K$ about the value of $\omega$ prior to the data being observed and $p(\omega \mid D, A, K)$ being the posterior probability density.

The following general description of BMA is primarily based on [44] and [45]. Suppose $\omega$ represents an input variable to a model. Its posterior distribution given data $\mathrm{D}$ is:

$$
\operatorname{pr}(\omega \mid D)=\sum_{k=1}^{K} \operatorname{pr}\left(\omega \mid M_{K}, D\right) \operatorname{pr}\left(M_{K} \mid D\right)
$$

where $M_{K}$ represents the considered models. This is an average of the posterior distributions und each of the models considered, weighted by their posterior model probabilities (PMPs). The posterior probability for model $M_{K}$ is given by the specific form of Bayes' theorem,

$$
\operatorname{pr}\left(M_{K} \mid D\right)=\frac{\operatorname{pr}\left(D \mid M_{K}\right) \operatorname{pr}\left(M_{K}\right)}{\sum_{l=1}^{K} \operatorname{pr}\left(D \mid M_{l}\right) \operatorname{pr}\left(M_{l}\right)}
$$

with

$$
\operatorname{pr}\left(D \mid M_{k}\right)=\int \operatorname{pr}\left(D \mid \theta_{k}, M_{k}\right) \operatorname{pr}\left(\theta_{k} \mid M_{k}\right) d \theta_{k}
$$

representing the integrated likelihood of model $M_{K^{*}} \cdot \theta_{k}$ is the vector of parameters of model $M_{K}, \operatorname{pr}\left(\theta_{k} \mid M_{k}\right)$ is the prior density of $\theta_{k}$ for model $M_{k}, \operatorname{pr}\left(D \mid \theta_{k}, M_{k}\right)$ is the likelihood and $\operatorname{pr}\left(M_{k}\right)$ is the prior probability that $M_{k}$ is the true model. For a regression model $\theta=\beta, \sigma^{2}$, all probabilities are implicitly conditional on the set of all models being considered.

Critique that has been offered for Bayesians includes but is not restricted to scepticism versus prior probabilities [46] and interpretational aspects $[47,48]$ and in response $[49,50]$. Some arguments are also briefly presented by Gelman [51]. It outreaches the possibilities within this text to discuss all of them; therefore, the focus will lie on critique related to Bayesian methods in the context of climate models and energy models. One of the main objections to the use of Bayesian methods is the arbitrariness of the prior distribution. In the context of climate science, Betz [46] argues that the dependence on (1) the specific prior probability distribution over the initially considered hypotheses and (2) the climate model used for probability estimates of climate sensitivity obtained by Bayesian learning is problematic. According to Betz, the choice of prior distribution is an arbitrary assumption and - in the context of climate modelling, with limited sample sizes - entail that the final posterior probability is a function of the initial prior (which is arbitrary). This critique of prior distribution influence on posterior probabilities is a well-known and not a new objection to Bayesian analysis cf. $[52,53]$.

Thus, the arbitrariness of the prior distribution is considered to be problematic. Put in Bayesian terms, an expert elicitation result is nothing but a collection of prior probabilities and though, this method is used for uncertainty assessment in climate modelling as well as energy modelling. If one accepts that a Bayesian statistician is an expert, the claim can be formulated even stronger, namely, that a Bayesian approach exactly satisfies requirement 4 (incorporate qualitative and quantitative aspects). This is to say that by means of prior distributions not only historical data (the likelihood) is used to assess uncertainty, but also a qualitative, subjective expert judgement can be incorporated. This not only renders the prior distribution choice a relevant tool for a complete representation but also responds to another critique that is often brought up against statistical methods in general, namely, that past evidence cannot provide for future developments. By means of a prior distribution, the likelihood of past events is relativized and both are possible, the recognition of the world as it is (was) and the representation of how this evidence is to be evaluated with respect to the future. It could be considered thus as a distinct virtue that prior probabilities depend on expert judgement rather than being problematic. The argument that subjective criteria can enrich a (statistical) model rather than disempower its findings due to lack of objectivism is also put forward by Isaac. His 'integrated subjectivism' also characterises a Bayesian model as the simplest form of integrating subjective knowledge and objective likelihoods with the aim of 'transforming a scientific model into a decision- 
theoretic one in which objective parameters (about the world) and subjective parameters (about the agent) peacefully coexist' [54].

Requirement 4 (incorporate qualitative and quantitative aspects, i.e. complete representation) is satisfied more explicitly with a BMA evaluation that uses informative priors. However, it has been argued that even improper priors (aka non-informative) or weak priors (i.e. flat priors) contain information about the subjective certainty of the modeller, e.g. [55].

Another criticism is sharpened by Kandlikar et al. [35] and focuses on two problematic assumptions:

- precision: the doctrine that uncertainty may be represented by a single probability or an unambiguously specified distribution;

- prior knowledge of sample space: the assumption that all possible outcomes (the sample space) and alternatives are known beforehand.

Indeed, the problem of deceptive preciseness of probability distributions needs to be addressed when an uncertainty assessment is based on probabilities. One mean to that end could be a transparent documentation of data used and assumptions made for the uncertainty assessment. Again, comparing with the predominant assessment method, expert elicitation, such critique could hold here as well, however, ambiguity in expert elicitation results seems to be perceived as less problematic. Another mean to that end could be a systematic sensitivity analysis in Bayesian terms. In this effort, a variety of prior probabilities and its effect on posterior probabilities could yield important insight, possibly even in cooperation with expert elicitation to define priors that are suitable ${ }^{k}$. BMA for input variables of energy models addresses this critique by evaluating a lower bound of uncertainty. Another possible way that is not investigated in the text could be the computation of interval probabilities that specify an interval of uncertainty for an input variable. However, due to considerations of ignorance, a lower bound seems more appropriate that respects the fact that unknown or intentionally ignored influences might increase uncertainty by a not specified amount.

Prior knowledge about the sample space $\Omega$ seems to pose more a problem in climate science than in energy economics. Possible outcomes and alternatives in energy economics are likely to be more predictable than in climate science. For example, in climate science, it might be true that a possible outcome is unknown due to interdependencies that are not well understood or orders of magnitude of effects that outrange expectations and the sample space does not account for that possibility. For example, if consequences of unprecedented gaseous concentrations (as in the past low $\mathrm{O}_{3}$ in the stratosphere [56] or more recently high $\mathrm{CO}_{2}$ concentrations in the atmosphere) are modelled, $\Omega$ might not be complete. In energy economics, some non-explicit assumptions such as that the target system will exist in a comparable way within the time horizon and geographic scope of the model will simplify the treatment and assessment of the sample space. This is not due to insufficient modelling techniques, but rather to science being an evolving matter that naturally develops with new insight, new measurement techniques and scientific understanding. However, the critique is certainly valid in the context of energy scenarios if key assumptions are considered such as gross domestic product (GDP) growth, future energy prices or population growth. Even if sound forecast data from statistical sources are available, these assumptions could be associated with deep uncertainty and possibly, the sample space $\Omega$ is not complete. This fact might belong to the realm of recognised ignorance, as Walker et al. term it. Especially for such key assumptions, an uncertainty assessment that evaluates as many potential influences on the key assumption as possible is adequate.

One possibility of limiting such deep uncertainty in the context of energy economic models is a deliberate choice of system boundaries. In addition to typically topological, economic or sectorial system boundaries and sub-system units, social systems can and should be detailed in energy models, see [57]. In energy models, as in climate models, one can intentionally define system boundaries to represent parts of the integrated (energy) system with simplified connections across the system boundaries. However, for climate models that are concerned with questions of global impact and consistent regional interpretation, meaningful results can only be obtained within a global system boundary. IPCC [58] specifies that only general circulation models (GCMs) have the potential of consistent estimates of regional climate change which are required in impact analysis. Energy models can be designed to depict a certain part of the global energy economic system, hereby possibly increasing uncertainty due to ignorance of effects on a larger scale, and possibly reducing uncertainty within the system boundaries as $\Omega$ becomes more complete. It thus seems to be a trade-off between chosen ignorance (due to system boundaries) and recognised ignorance (that one is aware of but cannot address). The BMA uncertainty assessment for input variables to energy models respects these uncertainties by formulating a lower bound of uncertainty.

It is worth discussing whether such uncertainties are better assessed with qualitative methods than in quantitative methods in probabilistic terms. The choice of key assumptions and their related uncertainty clearly limits the inferences that can be drawn from model results. However, the assessment of such deep uncertainties could be endeavoured in Bayesian terms. 


\section{The Bayesian endeavour}

A Bayesian approach could potentially satisfy the requirements previously defined. This section is concerned with how an implementation of Bayesian statistics for uncertainty assessment in energy models could be achieved. In Figure 4, a chart of the design of many quantitative energy models is shown. The information flow starts on the left side with influences that effect different input variables to energy models, exemplified by resources, demand and infrastructure for the input variable energy prices. Input variables are individual for every energy model so that the listed input variables energy prices, GDP, population, efficiency and demand can be regarded as typical examples. Input variables then are processed by the mathematical core of the energy model. Different types of models are possible; in the chart, the examples computable general equilibrium (CGE), linear programming (LP) models, mixed complementary problems (MCP) and stochastic models are mentioned. Finally, on the rightmost side, the output of the model, the energy scenario is the result of that information flow and computational effort.

The key idea is to assess the uncertainty of the input variables on the left side of the graph in Bayesian terms and thusly define a lower bound of uncertainties associated with model results (model output). If one accepts the premise that model output cannot be less uncertain than model input, this lower bound could be defined by the uncertainty of the input variables. It is important to stress at this point that the BMA method for input variables does not replace an energy model, e.g. LP, MCP or a CGE model, to name just a few that are a common practice in energy economics. The aim is rather to assess uncertainties of input variables that are specific for a given model by means of BMA. This process should render transparent that independent of the predictive power of an energy model the sheer use of variables that are inherently uncertain leads to model outcomes that must reflect that uncertainty. It can and should not be the aim of an energy model to present results as more or less certain than they are due to the nature of a nondeterministic world which the target system is based in. The structure, nature, scope, aim and mathematical formulation of energy models are highly diversified. For a given energy economic question, many different potential energy models can be designed to provide an answer. However, any model that could be designed will have input variables that are more or less uncertain. The aim of the proposed method is providing an estimation of these uncertainties independent of the specific (dis-) advantages a given model holds with respect to other energy models that could answer the question.

The predominant assessment method, expert elicitation, of uncertainty is used as reference. An expert elicitation process makes use of expert knowledge to assess how uncertain an assumption or a finding is. But what exactly is expert knowledge? The supposition is that expert knowledge is based on understanding of causal relationships, (long) record of observation or research, inclusion and exclusion of relevant factors and an intuitive 'feel' for the field of expertise. At least these virtues should be met by a Bayesian approach as well, together with the requirements previously defined.

The understanding of causal relationships - in the context of energy economics - refers to the ability of understanding market mechanisms, micro- and macroeconomic processes, social processes, etc. Consider the example of energy prices in Figure 4. If an assumption regarding the future energy price of, for example, natural gas is to be defined, it would be necessary to think of influences that impact the natural gas price, for example, resources, (global) demand, infrastructure, efficiency of devices and the like. These influences need not be assessed in qualitative terms or subjective opinion of an expert, for there are statistical data available. If such statistical data are not readily available, it might be necessary to look for a suitable statistical representation of the influence, e.g. for consumer acceptance [59], or methods described by [60] with respect to the food

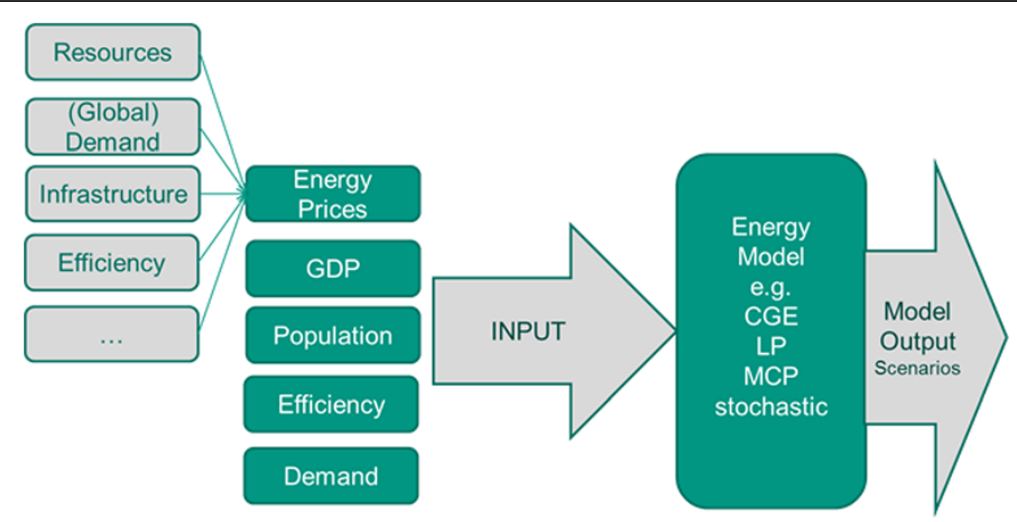

Figure 4 Schematic input-output structure of energy models. 
industry. A sound record of research and a long record of observation can be translated in statistical terms in sufficient large sample sizes of the statistical data. This might pose a problem if time series are short or the influence record is short.

The causal relationships, or how an influence bears on the input variable in question, in the example, the natural gas price, could be represented in a mathematical relation, e.g. a linear regression model. A regression model representing the dependent variable, natural gas price, and the explanatory variables, the influences, could capture causal relationships and the magnitude of impact of an influence on the input variable. Note that, non-linear models could be applied also, but for the analysis of the impact of an influence on the dependent variable (that is, the input variable in an energy model), it suffices to evaluate whether the influence increases or decreases the dependent variable and with what order of magnitude (that is, the coefficient estimate). This is straightforward standard statistical work. But this would not respect that the representation with a linear model itself increases uncertainty, for one might choose the wrong explanatory variables (influences) or not enough. This problem can be addressed by BMA.

BMA allows the inclusion and exclusion of potential influences by means of a Markov Chain Monte Carlo (MCMC) sampler ${ }^{\mathrm{m}}$ investigating the whole model space, i.e. the set of all possible variable combinations that can be employed to represent the dependent variable. In applying the BMA method, the uncertainty assessor firstly gathers any data that might be - even only in an indirect sense - be a relevant influence on the dependent variable. Let these candidate explanatory variables be $k$. The model space from which to choose the appropriate linear regression model is then $2^{\mathrm{k}}$. Any variable could be included or excluded, reliant on the explanatory value for the dependent variable. This explanatory value is assessed as posterior inclusion probability (PIP) for individual explanatory variables, and the individual models (containing specific explanatory variables) are ranked according to their PMP. Hence, the explanatory power of each variable and of different competing linear regression models can be assessed. As the name indicates, these results of BMA are probabilities. The prior probabilities concern the assessors' prior belief about how many explanatory variables are relevant. BMA then provides 1) the best linear regression model in terms of highest PMP and 2) the individual relevance of influences in terms of coefficient probability estimates and posterior inclusion probability PIP. In Figure 5, an exemplary coefficient estimate for an explanatory variable (GDP) of the natural gas price is illustrated.

On the abscissa, the coefficient value for the variable in the linear regression model is quantified. The ordinate represents the probability density for the coefficient

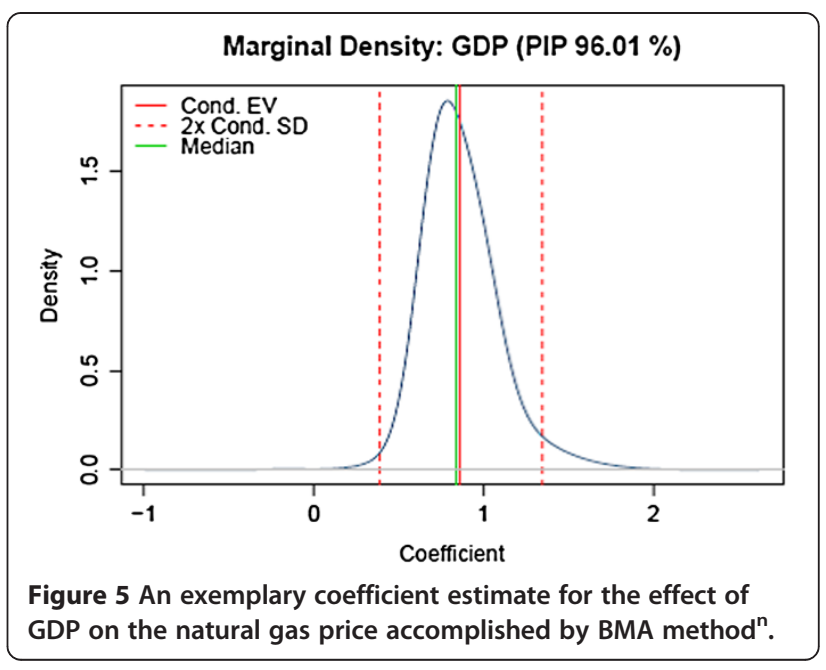

value (i.e. the rate of change of the conditional mean of the natural gas price conditional on the change of GDP). The double conditional standard deviation $(2 \times$ cond. SD) is indicated in the red dotted line. An equivalent chart can be produced for every explanatory variable of the competing models. The PIP of this variable is $96.1 \%$ what reflects that if the variable was contained in a model, competing models were less successful in explaining the data. In other words, the PIP is the sum of PMPs for all models wherein a covariate was included. The shape of the probability density and the low range of double standard deviation (approx. 0.4 to 1.4) indicate that variation from the conditional expected value (cond. EV) is rather low.

In practice the approach can be detailed in several steps. In step one, relevant input variables, or all input variables - depending on the size of the energy model under scrutiny - are identified, e.g. GDP ${ }^{\circ}$ within the energy models' system boundary. In the next step, statistical data of economic, ecological, social or from other disciplines is gathered that is suspected to influence the input variable (e.g. statistical data concerning, industrial production, import and export, taxes and subventions, birth and death rates, education, etc.), including statistical data of the input variable. This input variable (GDP) in the uncertainty assessment becomes the dependent variable on these influences. Note that, in contrast to other methods, there are hardly practical limitations to the amount of influences that can be considered, for BMA by means of a MCMC sampler investigates the model space and ranks explanatory variables (influences) according to their PIP. The next step is the definition of the form of mathematical representation, e.g. a multivariate linear regression ${ }^{\mathrm{P}}$. As many potential explanatory variables are defined, the question is what variables should be included in the model. BMA estimates models for all possible combinations of explanatory variables and constructs a weighted average over all of them. Then, the choice of 
a suitable prior distribution is defined, e.g. Zellner's gprior $[61,62]$. If the integrated likelihood is constant over all models, the PMP is proportional to the marginal likelihood of a specific candidate model, i.e. the probability of the data given that model times a prior probability. The prior probability reflects how probable the expert thinks the model is before looking at the data [63]. The thence generated models with highest PMPs can be evaluated, and a model that best represents the dependent variable (e.g. GDP) can be chosen. Finally, the uncertainty estimation for the input variable is derived from the PMP of the model chosen.

An additional feature that is not the focus of this text is the possibility of generating predictive distribution functions from the chosen model that consistently with past evidence and expert judgement represent the dependent variable for given assumptions of explanatory variables. This could foster consistency in the choice of key assumptions.

The interpretation of BMA results as uncertainty can be straight forward if uncertainty is suitable defined. To that end, a definition that is based on probability is introduced.

Definition: Uncertainty equals the probability that statement $S$ might not be true.

Given, by means of BMA, a PMP is calculated for an uncertainty model (e.g. a PMP of $13 \%{ }^{\mathrm{q}}$ for a model that represents the natural gas price), uncertainty - by definition - would be at least $87 \%$ for the dependent variable. This would mean that the input variable 'natural gas price' to an energy model holds an uncertainty of at least $87 \%$, even if all relevant explanatory variables are considered. Hence, the results of a model including an assumption about the natural gas price cannot be less uncertain than $87 \%$.

In other words, the PMP reflects the probability that the input variable thusly described matches data. For a model with a PMP of $13 \%$, the associated uncertainty would be at least $87 \%$. A clarifying statement of the following form could accompany model results.

"In consideration of expert judgement, statistical data of influence $\mathrm{X}_{1}$, influence $X_{2}$, influence $\mathrm{X}_{3}, \ldots$, of the last 25 years, the uncertainty that the input variable can be described as such is at least $87 \%$."

For every influence $\mathrm{X}_{1}, X_{2}, \ldots$, the PIP indicates the explanatory contribution of the influence and 1-PIP indicates the uncertainty that the influence contributes to explaining the dependent variable of a given model (typically the one with the highest PMP). In the example, the uncertainty that GDP explains the natural gas price (together with the other explanatory variables) of the chosen model is $3.99 \%(1-0.9601=0.0399)$. Such an assessment clearly satisfies requirement 1 as uncertainty expressed as probability density is a clear indication how reliable the findings are.
The third virtue of expert knowledge, inclusion and exclusion of relevant factors, could be achieved by this standardised method, hereby satisfying requirement 2 (applicable independent of assessor's expertise).

The approach would limit many intuitive over- or under-estimations of impact of influences on variables that figure as input variables in energy models. It is thinkable that different experts evaluate individual influences as more/less relevant for the assumption of an input variable (e.g. a natural gas price assumption) thereby generating ambiguousness and dissent. A standardised method, relying on statistical data, i.e. knowledge with little associated uncertainty in and by itself, could yield significant improvement in uncertainty assessment for energy models. However, as expert knowledge is an important part of assessment methods, it is possible to take this by prior probability specification into account.

A key quality of the BMA method for input variables is that model uncertainty of the linear regression model itself, and thus, the assessment method's uncertainty is quantified in probabilistic terms. This is a distinct advantage of the method as opposed to purely statistical or qualitative methods. Other methods that are applied in uncertainty analysis, for example, standard statistical analysis or purely qualitative methods ignore that source of uncertainty. A standard regression analysis is conditional on the assumed statistical model, and the analyst may be uncertain whether it is the best representation. If an expert Delphi $[64,65]$ is carried out opinions are rarely scrutinised for their correctness or compliance with statistical evidence. However, if an expert is asked, how probable she thinks her evaluation is, a prior distribution could be constructed.

Another requirement previously defined is the applicability to different energy models (comparability of results), requirement 3 . As indicated by Figure 4, the assessment method is concerned with input data to energy models and is hence independent of the mathematical model that consequently processes the input. The uncertainty assessment method would be applicable for different kinds of models common in energy economics, LP's, MCP's, CGE's, stochastic models or even qualitative models that use input variables.

Requirement 4 (inclusion of qualitative and quantitative aspects) to assure a complete representation can be achieved through prior probabilities and statistical data. The resulting posterior probabilities and the probabilistic interpretation of uncertainty are straightforward to communicate, as demanded in requirement 5 (intuitively understandable and straightforward to communicate).

Finally, requirement 6 demands for reproducibility and unambiguousness. Given assessors use the same set of data, the results of BMA are reproducible. However, a source of ambiguousness could be prior probability 
choice. This lies, as previously discussed, in the very nature of expert judgement. A sensitivity analysis to evaluate such ambiguousness could both, increase understanding of the BMA method within this context, and indicate to what extent expert elicitation has to be put in perspective to statistical data.

\section{Results and discussion}

Results of applying BMA to energy model input variables are PMPs of competing models for input variables of an energy model. The PMPs of the input variables can then be used to define quantitatively the associated uncertainty of the specific input variable. The method respects previously defined requirements. The result is an uncertainty assessment of the form: applied input variable $X$ has an associated uncertainty of at least $Y \%$. Results of the model are thus associated with an uncertainty of at least $Y \%$. Note, that such a result demands acceptance of the premise that model results cannot be less uncertain than model input.

Existing uncertainty assessments for energy models provide evaluations of energy models or energy scenarios. However, the approaches discussed in this text lack some qualities in the context of energy modelling that BMA for input variable uncertainty estimation could provide.

The method described by Walker et al. is rather a classification of uncertainty than an assessment that explicitly states uncertainty of results (requirement 1). On the other hand, methods that are applied in classical uncertainty quantification ${ }^{\mathrm{r}}$ such as statistical analysis, stochastic modelling or error propagation computation, although being explicit, treat uncertainties in a mechanistic way that does not respect the various social and political aspects (requirement 4). Methods that mainly rely on expert elicitation, such as the NUSAP method might lack reproducibility of results and objectiveness (requirements 2 and 6). BMA could potentially combine the desired qualities. Intuitively understandable (requirement 5) uncertainty assessments that can be produced for different energy models (requirement 3 ) only dependent on the respective input variables the model demands could provide relevant insight in uncertainties that are associated with model input. As potential consequence of applying BMA for input variable uncertainty, transparency regarding model results with respect to the reliability of such findings could be evaluated and communicated. Moreover, input variables could be classified according to their adherent uncertainty if the method is applied. And finally, but left for further research, the possibility of generating predictive densities by means of BMA could lead to consistent input variable values that respect influences across system boundaries of a specific model.

All uncertainty assessment methods have advantages and disadvantages. In spite of the successful fulfilment of previously defined requirements, the BMA approach for input variables has deficits that need to be discussed.

A rather practical issue stems from the fact that the approach is parametric. This means that in practice, many different input variables need to be assessed if large and complex models are analysed and a significant amount of data collection and preparation seems necessary. One way, which proved successful in the NUSAP method, for reduction of assessment variables is a classification of input variables and a consequent sensitivity analysis to discern highly relevant input variables [25]. Such a procedure could be suitable for the BMA approach as well.

Another issue might arise if input variables yield individual uncertainties of different orders of magnitude. The question then arises whether the least certain defines the uncertainty or if model dependent interpretation of individual uncertainties (of individual input variables) would be meaningful. It is not straightforward to see where in the mathematical core of energy models input variables are processed, and hence, tracing back results to individual inputs could be difficult. A form of meta-analysis, as proposed by $[66,67]$ could possibly give relevant insight regarding the uncertainty significance of individual input variables across studies of different model applications, as done for studies in medicine (psychotherapy) [68].

And finally, an issue could arise if an energy model incorporates aspects or effects that are relatively 'new', e.g. unconventional gas in Europe. Due to data scarcity and lacking maturity of available processes, a Bayesian approach to assess such input data would be difficult. The same problem of data scarcity can occur if scenario assumptions are not explicit, e.g. social, or psychological assumptions. If data are available for such assumptions, their bearing on an input variable to an energy model can be incorporated by the BMA method and hence could increase transparency in that aspect. If data are not available, it must be communicated that the aspect is not part of the uncertainty assessment.

In the light of increased transparency, inter-subjective independency, quantitative explicit results, comparability of results and methodological advantages (reproducibility and inclusion of subjective expert judgements), the expected value of assessing input variable uncertainty of energy models amounts to a better understanding of the associated uncertainty of an energy scenario. This is valuable information for the evaluation of results of different models. For example, top-down models are distinct from bottom-up models with regard to the assumptions they apply. The proposed uncertainty assessment could potentially add value for the comparison of energy scenarios that stem from different models. In addition, but left to further research, is the potential of scrutinising model ensembles ${ }^{\mathrm{s}}$, as demonstrated by [69] in the context of weather forecasts. 


\section{Conclusions}

BMA for uncertainty quantification of input variables could potentially satisfy the requirements that a versatile applicable and standardised method for uncertainty assessment in energy economic modelling demands. Given the described advantages and disadvantages, it is at least worth discussion whether such an approach could improve the assessment itself and consequently could put the inferences and policy recommendations based on model results in perspective. This in turn should enable stakeholders and decision makers to include reported uncertainties in their decision making processes and increase trust in scientific findings. Trust in scientific findings is not solely generated by unerring model results but also by acknowledgement and transparency of uncertainties respecting that reality is not strictly deterministic.

Further research should be undertaken concerning the critical remarks and potential solutions for the application of the method. To this end, firstly an application of the approach to different models should yield insight allowing for further improvement of the approach.

\section{Endnotes}

${ }^{\mathrm{a}}$ For further information on energy models as referred to in this text see [70-72], or [73] in Germany.

${ }^{\mathrm{b}}$ The green kite is spanned up by the minimum scores in each group for each pedigree criterion; the orange kite is spanned up by the maximum scores. The orange band between the green kite and the red area represents expert disagreement on the pedigree scores for that variable. In some cases, the picture was strongly influenced by a single deviating low score given by one of the six experts. In those cases, the light green kite shows what the green kite would look like if that outlier would have been omitted.

${ }^{\mathrm{c}}$ Given that the same experts evaluate many fields of model-associated uncertainties, it is thinkable that the expertise in some areas is not as sound as one would expect.

${ }^{\mathrm{d}}$ e.g. Limited number of experts, limited knowledge of experts.

${ }^{\mathrm{e}}$ However, Smithson [74] has made a strong case that 'in all tasks, precise-conflictive sources were viewed as less credible than ambiguous-consensual ones even when subjects expressed preference for the preciseconflictive alternative.' what suggests that the requirement of inter-subjectivity has more relevance in terms of acceptance of the assessment.

${ }^{\mathrm{f}}$ This might trace back to a criticism of [35] on the interdependence of likelihood and confidence: 'When an event is said to be extremely likely (or extremely unlikely) it is implicit that we have high confidence'.

${ }^{\mathrm{g}}$ As defined by [35], uncertainty that results from myriad factors both scientific and social and consequently is difficult to accurately define and quantify.
${ }^{\mathrm{h}}$ Qualification of the degree of agreement: summary terms: low, medium or high [29].

${ }^{\mathrm{i}}$ By that is meant that the uncertainty captured between 2 and 3 is not more/less than the uncertainty captured between 4 and 5 , or for that matter 5 and 6 .

${ }^{j}$ For more information see for example [75-77].

${ }^{k}$ e.g. Experts could be questioned what probability to a qualitative assessment like 'surely no more/less than' can be attributed. In that way, a subjective degree of belief can lead to a subjective prior distribution. See also $[78,79]$.

${ }^{\mathrm{I}}$ Such as $[80,81]$.

${ }^{\mathrm{m}}$ One could also use a Bayesian Markov chain Monte Carlo (BMCMC), as for example, Kim et al. have done to determine optimum tender prices [82].

${ }^{\mathrm{n}}$ This result stems from work not published yet, available from the author. Abbreviations: GDP gross domestic product, PIP posterior inclusion probability, Cond. EV Conditional expected value, SD standard deviation. Note that, the shape of the probability distribution offers a further indication of the reliability of the conditional expected value.

${ }^{\circ}$ Note that, input variables may vary considerably between models. For example, a bottom up model as the TIMES linear program [2] does not enter economic performance directly in form of a GDP input variable. Instead, such macro-economic assumptions must be translated in sectorial demands, e.g. megakilogrammes of crude steel demand. This transformation is often done based solely on expert judgement for a given sector of an energy model. A BMA uncertainty assessment could improve transparency with regard to that process. An example of a demand forecast is industrial production is provided by [83].

${ }^{\mathrm{P}}$ Of course other models can be applied as well, for example, generalised linear models, proportional hazard models or logistic regressions.

${ }^{\mathrm{q}} \mathrm{A}$ posterior model probability (PMP) of ca. $11 \%$ is a rather poor model representation of observed data. However, similar approaches in other contexts show that low PMPs are not unusual, e.g. infrastructure PMP 0.39 [84], econometric context PMP 0.3 [85], medical context PMP 0.17 in a dataset on primary biliary cirrhosis [44] or [86]. For an explicit application to forecasts, see [69]. For a BMA example in the context of hydrology, where the BMA method was coupled with a maximum likelihood estimation proposed by Taplin, see [87].

${ }^{\mathrm{r}}$ Mainly applied in engineering.

${ }^{s}$ Ensembles as used by Raftery are model results in which a model is run several times with different initial conditions or model physics. This might be applicable for energy models as well where different key assumptions are applied or key assumptions are varied. 


\section{Competing interests}

The author is a funded member of the Helmholtz Research School on Energy Scenarios.

\section{Acknowledgements}

The author Monika Culka would like to thank the reviewers for their comments that helped improving the manuscript.

Received: 2 May 2014 Accepted: 30 September 2014

Published online: 24 October 2014

\section{References}

1. Pollitt H (2014) E3ME Technical Manual, Version 6.0 Cambridge Econometrics. Online http://www.camecon.com/Libraries/ Downloadable_Files/E3ME_Manual.sflb.ashx

2. Loulou R, Remme U, Kanudia A, Lehtila A, Goldstein G (2005) Documentation for the TIMES Model. PART I. IEA ETSAP. Online: www.iea-etsap.org

3. Meerschaert MM (2013) Stochastic models. In: Mathematical Modeling: Elsevier. S. Academic, Boston, pp 251-299

4. Wallace SW, Fleten S (2003) Stochastic programming models in energy. In: Stochastic Programming, vol 10. Handbooks in Operations Research and Management Science. Elsevier, pp 637-677

5. Gabriel SA (2010) Complementarity Modeling in Energy Markets. International Series in Operations Research \& Management Science, vol 2150. Springer, New York, London

6. Dieckhoff C (2011) Energieszenarien. Konstruktion, Bewertung und Wirkung - "Anbieter" und "Nachfrager" im Dialog. Energieszenarien. KIT Scientific Publishing, Karlsruhe

7. Grunwald A (2011) Energy futures: diversity and the need for assessment. Futures 43(8):820-830. doi:10.1016/j.futures.2011.05.024

8. Bundesregierung Deutschland (2010) Energiekonzept der Bundesregierung vom. September 2010. Online: http://www.bundesregierung.de

9. Commission E (2012) Energy Roadmap 2050. Energy. Publications Office of the European Union, Luxembourg

10. EWI GWS PROGNOS (2010) Energieszenarien für ein Energiekonzept der Bundesregierung. Projekt Nr. 12/10 des Bundesministeriums für Wirtschaft und Technologie, Basel/Köln/Osnabrück

11. Weimer-Jehle W (2006) Cross-impact balances: a system-theoretical approach to cross-impact analysis. Technol Forecast Soc Change 73(4):334-361. doi:10.1016/j.techfore.2005.06.005

12. Walker WE, Harremoes $P$, Rotmans J, van der Sluijs JP, van Asselt MBA, Janssen P, Krayer von Krauss MP (2005) Defining uncertainty: a conceptual basis for uncertainty management in model-based decision support. Integr Assessment 4(1). doi:10.1076/iaij.4.1.5.16466

13. Michaelides PG, Fassois SD (2013) Experimental identification of structural uncertainty - an assessment of conventional and non-conventional stochastic identification techniques. Eng Struct 53:112-121. doi:10.1016/j. engstruct.2013.03.033

14. Kovacevic RM, Paraschiv F (2014) Medium-term planning for thermal electricity production. OR Spectrum 36(3):723-759. doi:10.1007/s00291-013-0340-9

15. Sura P (2003) Stochastic analysis of southern and pacific ocean Sea surface winds. J Atmos Sci 60(4):654-666. doi:10.1175/1520-0469(2003)060<0654: SAOSAP $>2.0 . \mathrm{CO} ; 2$

16. Zavala-Garay J, Moore AM, Perez CL, Kleeman R (2003) The response of a coupled model of ENSO to observed estimates of stochastic forcing. J Climate 16(17):2827-2842. doi:10.1175/1520-0442(2003)016<2827: TROACM>2.0.CO;2

17. Smith RC (2013) Uncertainty Quantification. Theory, Implementation, and Applications. Computational Science \& Engineering Series. SIAM-Society for Industrial and Applied Mathematics, PA USA

18. Oreskes N, Shrader-Frechette K, Belitz K (1994) Verification, validation, and confirmation of numerical models in the earth sciences. Science 263 (5147):641-646

19. Betz G (2008) Der Umgang Mit Zukunftswissen in der Klimapolitikberatung Eine Fallstudie Zum stern review. Philos Nat 45(1):95-129

20. Lloyd EA (2010) Confirmation and robustness of climate models. Philos Sci 77(5):971-984

21. Parker WS (2009) II-confirmation and adequacy-for-purpose in climate modelling. Aristotelian Soc Suppl 83(1):233-249. doi:10.1111/j.14678349.2009.00180.x
22. Mastrandrea MD, Mach KJ (2011) Treatment of uncertainties in IPCC assessment reports: past approaches and considerations for the fifth assessment report. Clim Change 108(4):659-673. doi:10.1007/s10584-011-0177-7

23. Mastrandrea MD, Field CB, Stocker TF, Edenhofer O, Ebi KL, Frame DJ, Held H, Kriegler E, Mach KJ, Matschoss PR, Plattner GK, Yohe G (2011) The IPCC AR5 guidance note on consistent treatment of uncertainties: a common approach across the working groups. Clim Change 108(4):675-691. doi:10.1007/s10584-011-0178-6

24. Barrett S, Dannenberg A (2012) Climate negotiations under scientific uncertainty. Proc Natl Acad Sci USA 109(43):17372-17376. doi:10.1073/ pnas. 1208417109

25. van der Sluijs JP, Potting J, Risbey J, van Vuuren D, de Vries B, Beusen A, Heuberger P, Corral Quintana S, Funtowicz S, Kloprogge P, Nujiten D, Petersen A, Ravetz J (2002) Uncertainty assessment of the IMAGE/TIMER B1 CO2 emissions scenario, using the NUSAP method, Report No: 410200104

26. Rai V (2013) Expert elicitation methods for studying technological change under uncertainty. Environ Res Lett 8(4):041003. doi:10.1088/1748-9326/8/4/ 041003

27. Le Maitre OP, Knio OM (2010) Spectral Methods for Uncertainty Quantification with Applications to Computational Fluid Dynamics. Scientific computation, Springer, Dordrecht, New York

28. Council IA (2010) Climate Change Assessments. Review of the Processes and Procedures of the IPCC. Inter Academy Council, Amsterdam, The Netherlands

29. Mastrandrea MD, Field CB, Stocker TF, Edenhofer O, Ebi KL, Frame DJ, Held H, Kriegler E, Mach KJ, Matschoss PR, Plattner GK, Yohe GW, Zwiers FW (2010) Guidance Note for Lead Authors of the IPCC Fifth Assessment Report on Consistent Treatment of Uncertainties. Intergovernmental Panel on Climate Change (IPCC), CA, USA

30. Krueger T, Page T, Hubacek K, Smith L, Hiscock K (2012) The role of expert opinion in environmental modelling. Environ Model Software 36:4-18. doi:10.1016/j.envsoft.2012.01.011

31. Der Sluijs V, Jeroen P, Craye M, Funtowicz S, Kloprogge P, Ravetz J, Risbey J (2005) Combining quantitative and qualitative measures of uncertainty in model-based environmental assessment: the NUSAP system. Risk Anal 25 (2):481-492. doi:10.1111/j.1539-6924.2005.00604.x

32. Aven T, Pörn K (1998) Expressing and interpreting the results of quantitative risk analyses. Rev Discuss Reliability Eng Syst Saf 61(1-2):3-10. doi:10.1016/ S0951-8320(97)00060-4

33. Jaeger C (2001) Risk, Uncertainty, and Rational Action. Risk, Society, and Policy Series. Earthscan, London

34. Patt A, Dessai S (2005) Communicating uncertainty: lessons learned and suggestions for climate change assessment. Comptes Rendus Geoscience 337(4):425-441. doi:10.1016/j.crte.2004.10.004

35. Kandlikar M, Risbey J, Dessai S (2005) Representing and communicating deep uncertainty in climate-change assessments. Comptes Rendus Geoscience 337(4):443-455. doi:10.1016/j.crte.2004.10.010

36. Pilavachi PA, Dalamaga T, Rossetti di Valdalbero D, Guilmot JF (2008) Ex-post evaluation of European energy models. Energy Policy 36(5):1726-1735. doi:10.1016/j.enpol.2008.01.028

37. Bezdek RH, Wendling RM (2002) A half century of long-range energy forecasts: errors made, lessons learned, and implications for forecasting. J Fusion Energ 21(3-4):155-172. doi:10.1023/A:1026208113925

38. Knutti R, Furrer R, Tebaldi C, Cermak J, Meehl GA (2010) Challenges in combining projections from multiple climate models. J Climate 23 (10):2739-2758. doi:10.1175/2009JCLI3361.1

39. Weisberg M (2006) Robustness analysis. Philos Sci 73(5):730-742

40. Gent PR, Danabasoglu G, Donner L, Holland MM, Hunke EC, Jayne SR, Lawrence DM, Neale RB, Rasch PJ, Vertenstein M, Worley PH, Yang ZL, Zhang M (2011) The community climate system model version 4. J Climate 24(19):4973-4991. doi:10.1175/2011JCLI4083.1

41. Stevens B, Bony S (2013) What are climate models missing? Science 340 (6136):1053-1054. doi:10.1126/science.1237554

42. Hughes RIG (1993) A Philosophical Companion to First-Order Logic. Hackett Pub. Co., Indianapolis

43. Bernardo JM (2003) Probability and Statistics. In: Bernardo JM (ed) Encyclopedia of Life Support Systems (EOLSS). Bayesian Statistics. Eolss Publishers, Paris

44. Hoeting JA, Madigan D, Raftery AE, Volinsky CT (1999) Bayesian model averaging: a tutorial. Stat Sci 382-401

45. Raftery AE, Madigan D, Hoeting JA (1997) Bayesian model averaging for linear regression models. J Am Stat Assoc 92(437):179-191 
46. Betz G (2007) Probabilities in climate policy advice: a critical comment. Clim Change 85(1-2):1-9

47. Thompson B (2007) A critique of Bayesian inference. In: The Nature of Statistical Evidence, vol 189. Springer, New York, pp 84-96

48. Gelman A, Shalizi CR (2013) Philosophy and the practice of Bayesian statistics. Br J Math Stat Psychol 66(1):8-38. doi:10.1111/j.2044 8317.2011.02037.x

49. Mayo DG (2013) The error-statistical philosophy and the practice of Bayesian statistics: comments on Gelman and Shalizi: 'Philosophy and the practice of Bayesian statistics'. Br J Math Stat Psychol 66(1):57-64. doi:10.1111/j.20448317.2012.02064.x

50. Burstyn I, Kromhout H (2002) A critique of Bayesian methods for retrospective exposure assessment. Ann Occup Hyg 46(4):429-431. doi:10.1093/annhyg/mef058

51. Gelman A (2008) Objections to Bayesian statistics. Bayesian Anal 3:445-450 doi:10.1214/08-BA318

52. Winkler RL (1967) The assessment of prior distributions in Bayesian analysis. J Am Stat Assoc 62(319):776. doi:10.2307/2283671

53. Pierce DA, Folks $J$ (1969) Sensitivity of Bayes procedures to the prior distribution. Oper Res 17(2):344-350. doi:10.1287/opre.17.2.344

54. Isaac AM (2014) Model uncertainty and policy choice: a plea for integrated subjectivism. Stud Hist Philos Sci Part A 47:42-50. doi:10.1016/j. shpsa.2014.05.004

55. van Dongen S (2006) Prior specification in Bayesian statistics: three cautionary tales. J Theor Biol 242(1):90-100. doi:10.1016/j.jtbi.2006.02.002

56. Stolarski R, Bojkov R, Bishop L, Zerefos C, Staehelin J, Zawodny J (1992) Measured trends in stratospheric ozone. Science 256(5055):342-349

57. Luhmann N (1995) Social Systems. Stanford University Press, Stanford, Calif, Writing science

58. Intergovernmental Panel on Climate Change (2013) What is a GCM? Guidance on the Use of Data. Online: www.ipcc-data.org

59. Strickert DP (1990) Estimating consumer acceptance limits. Commun Stat Theory Methods 19(7):2365-2472. doi:10.1080/03610929008830327

60. Næs T, Brockhoff PB, Tomić O (2010) Statistics for sensory and consumer science. Wiley, Chichester, West Sussex, Hoboken, NJ

61. De Finetti B, Goel Prem K, Zellner A (1986) Bayesian Inference and Decision Techniques. Essays in Honor of Bruno de Finetti. North-Holland, Sole distributors for the USA and Canada, Elsevier Science Pub. Co, Amsterdam, New York, New York, NY, USA

62. Zellner A, Hong C (1989) Forecasting international growth rates using Bayesian shrinkage and other procedures. J Econom 40(1):183-202. doi:10.1016/0304-4076(89)90036-5

63. Zeugner S (2011) Bayesian Model Averaging with BMS for BMS version 0.3.0. Online: www.bms.zeugner.eu

64. Adler M, Ziglio E (1996) Gazing into the Oracle. The Delphi Method and its Application to Social Policy and Public Health. Jessica Kingsley Publishers, London

65. Ayyub BM (2001) Elicitation of expert opinions for uncertainty and risks. CRC Press, Boca Raton, Fla

66. Glass GV (1977) 9: integrating findings: the meta-analysis of research. Rev Res Educ 5(1):351-379. doi:10.3102/0091732X005001351

67. Hedges LV (1982) Statistical Methodology in Meta-Analysis. ERIC Clearinghouse on Tests, Measurement, and Evaluation, Princeton, NJ

68. Smith ML, Glass GV (1977) Meta-analysis of psychotherapy outcome studies. Am Psychol 32(9):752-760. doi:10.1037/0003-066X.32.9.752

69. Raftery AE, Gneiting T, Balabdaoui F, Polakowski M (2005) Using Bayesian model averaging to calibrate forecast ensembles. Mon Weather Rev 133 (5):1155-1174. doi:10.1175/MWR2906.

70. Capros P, Paroussos L, Fragkos P, Tsani S, Boitier B, Wagner F, Busch S, Resch G, Blesl M, Bollen J (2014) Description of models and scenarios used to assess European decarbonisation pathways. Energ Strategy Rev 2(3-4):220-230. doi:10.1016/j.esr.2013.12.008

71. Jebaraj S, Iniyan S (2006) A review of energy models. Renew Sustain Energ Rev 10(4):281-311. doi:10.1016/j.rser.2004.09.004

72. Bhattacharyya SC, Timilsina GR (2010) A review of energy system models. Int J Energ Sector Manage 4(4):494-518. doi:10.1108/17506221011092742

73. Fahl U, Forum für Energiemodelle und Energiewirtschaftliche System analysen in Deutschland (2004) Energiemodelle zum Klimaschutz in liberalisierten Energiemärkten: die Rolle erneuerbarer Energieträger. Umwelt- und Ressourcenökonomik, LIT
74. Smithson M (1999) Conflict aversion: preference for ambiguity vs conflict in sources and evidence. Organ Behav Hum Decis Process 79(3):179-198. doi:10.1006/obhd.1999.2844

75. Community Earth System Model CESM (2014) Models. CO, USA

76. Program for Climate Model Diagnosis and Intercomparison (2013) About the WCRP CMIP3 Multi-Model Dataset Archive at PCMDI. Online: http://www-pcmdi.llnl.gov/

77. Meehl GA, Covey C, Taylor KE, Delworth T, Stouffer RJ, Latif M, McAvaney B, Mitchell JFB (2007) THE WCRP CMIP3 Multimodel dataset: a new era in climate change research. Bull Am Meteorol Soc 88(9):1383-1394. doi:10.1175/BAMS-88-9-1383

78. Eicher TS, Papageorgiou C, Raftery AE (2011) Default priors and predictive performance in Bayesian model averaging, with application to growth determinants. J Appl Econ 26(1):30-55

79. Ley E, Steel MF (2012) Mixtures of -priors for Bayesian model averaging with economic applications. J Econom 171(2):251-266. doi:10.1016/j. jeconom.2012.06.009

80. Department of Economic and Social Affairs, Population Division (2013) World Population Prospects: The 2012 Revision, Highlights and Advance Tables. New York. Working Paper No. ESA/P/WP. 228

81. OECD (2012) Main Economic Indicators. OECD Publishing, Main Economic Indicators - complete database,

82. Kim S, Kim G, Lee D (2014) Bayesian Markov chain Monte Carlo model for determining optimum tender price in multifamily housing projects. J Comput Civ Eng 28(3):06014001. doi:10.1061/(ASCE)CP.1943-5487.0000297

83. Feldkircher M (2012) Forecast combination and Bayesian model averaging: a prior sensitivity analysis. J Forecast 31(4):361-376. doi:10.1002/for.1228

84. Wesonga R (2014) Bayesian model averaging: an application to the determinants of airport departure delay in Uganda. AJTAS 3(1):1 doi: 10.11648/j.ajtas.20140301.11

85. Fernandez C, Ley E, Steel MFJ (2001) Model uncertainty in cross-country growth regressions. J Appl Econ 16(5):563-576. doi:10.1002/jae.608

86. Chua CL, Suardi S, Tsiaplias S (2013) Predicting short-term interest rates using Bayesian model averaging: evidence from weekly and high frequency data. Int J Forecast 29(3):442-455

87. Neuman SP (2003) Maximum likelihood Bayesian averaging of uncertain model predictions. Stoch Environ Res Risk Assess 17(5):291-305. doi:10.1007/ s00477-003-0151-7

\section{doi:10.1186/s13705-014-0021-9}

Cite this article as: Culka: Applying Bayesian model averaging for uncertainty estimation of input data in energy modelling. Energy, Sustainability and Society 2014 4:21.

\section{Submit your manuscript to a SpringerOpen ${ }^{\odot}$ journal and benefit from:}

- Convenient online submission

- Rigorous peer review

- Immediate publication on acceptance

- Open access: articles freely available online

- High visibility within the field

- Retaining the copyright to your article

Submit your next manuscript at springeropen.com 